\title{
Polypharmacy and Hyperpolypharmacy in Older Individuals with Parkinson's Disease: A Systematic Review and Meta-Analysis
}

\author{
Akshaya Srikanth Bhagavathula ${ }^{a}$ Wubshet Tesfaye ${ }^{b} \quad$ Kota Vidyasagar $^{c}$ \\ Daniela Fialova ${ }^{\mathrm{a}, \mathrm{d}}$ \\ aDepartment of Social and Clinical Pharmacy, Faculty of Pharmacy in Hradec Kralove, Charles University, \\ Hradec Kralove, Czech Republic; ${ }^{b}$ Health Research Institute, University of Canberra, Canberra, ACT, Australia; \\ 'University College of Pharmaceutical Sciences, Kakatiya University, Warangal, India; ${ }^{d}$ Department of \\ Geriatrics and Gerontology, 1st Faculty of Medicine, Charles University, Prague, Czech Republic
}

\section{Keywords}

Polypharmacy · Hyperpolypharmacy · Parkinson's disease ·

Cognitive function - Meta-analysis

\begin{abstract}
Background and Aim: Polypharmacy (concomitant use of 5-9 medicines) and hyperpolypharmacy (concomitant use of over 10 medicines) were observed to be more frequent in older adults ( $\geq 65$ years) and associated with adverse outcomes. Their prevalence and risk in older patients with Parkinson's disease (PD) remain unknown. We aimed to synthesize the extant evidence on the prevalence and risk of polypharmacy and hyperpolypharmacy in older adults with PD. Methods: A systematic literature search was performed in PubMed/MEDLINE, Scopus, and Embase databases to identify pertinent studies published from 2000 to July 2021. Observational studies reporting the prevalence and association with disease of polypharmacy/hyperpolypharmacy in older adults with PD were meta-analyzed. Pooled prevalence and odds ratio (OR) with $95 \%$ confidence intervals (Cls) were calculated. Results: Out of the total 499 studies identified, 6 fulfilled the inclusion criteria and comprised 7,171 participants. The overall prevalence of polypharmacy and hyperpoly-
\end{abstract}

karger@karger.com www.karger.com/ger

Karger!"

BOPEN ACCESS
(C) 2022 The Author(s)

Published by S. Karger AG, Basel

This is an Open Access article licensed under the Creative Commons Attribution-NonCommercial-4.0 International License (CC BY-NC) (http://www.karger.com/Services/OpenAccessLicense), applicable to the online version of the article only. Usage and distribution for commercial purposes requires written permission. pharmacy was 40\% (95\% Cl: 37-44) and 18\% (95\% Cl: 13-23), respectively. A meta-analysis of 4 studies indicated a significant association between polypharmacy (OR: 1.94, 95\% Cl: $1.26-2.62 ; p<0.001)$ and PD. Hyperpolypharmacy was also strongly associated with PD (OR: 3.11,95\% Cl: 2.08-4.14; $p<$ 0.001). Conclusion: Polypharmacy (40\%) and hyperpolypharmacy $(18 \%)$ are highly prevalent and eventually increase the risk of drug-related problems in older adults with PD. Therefore, interventions that ensure rational geriatric pharmacotherapy are of critical importance for the older population with neurogenerative disorders.

(C) 2022 The Author(s).

Published by S. Karger AG, Basel

\section{Introduction}

Parkinson's disease (PD) is a chronic neurodegenerative disease characterized by progressive slowness of movement (bradykinesia) along with motor symptoms like rest tremor, rigidity, and postural instability [1]. PD is among the highly prevalent neurodegenerative disorders that nearly doubled in prevalence over the past few decades mainly due to the increasing older population across the globe and is precipitated by contributions from 
Fig. 1. PRISMA 2020 diagram of the literature selection in this systematic literature review.

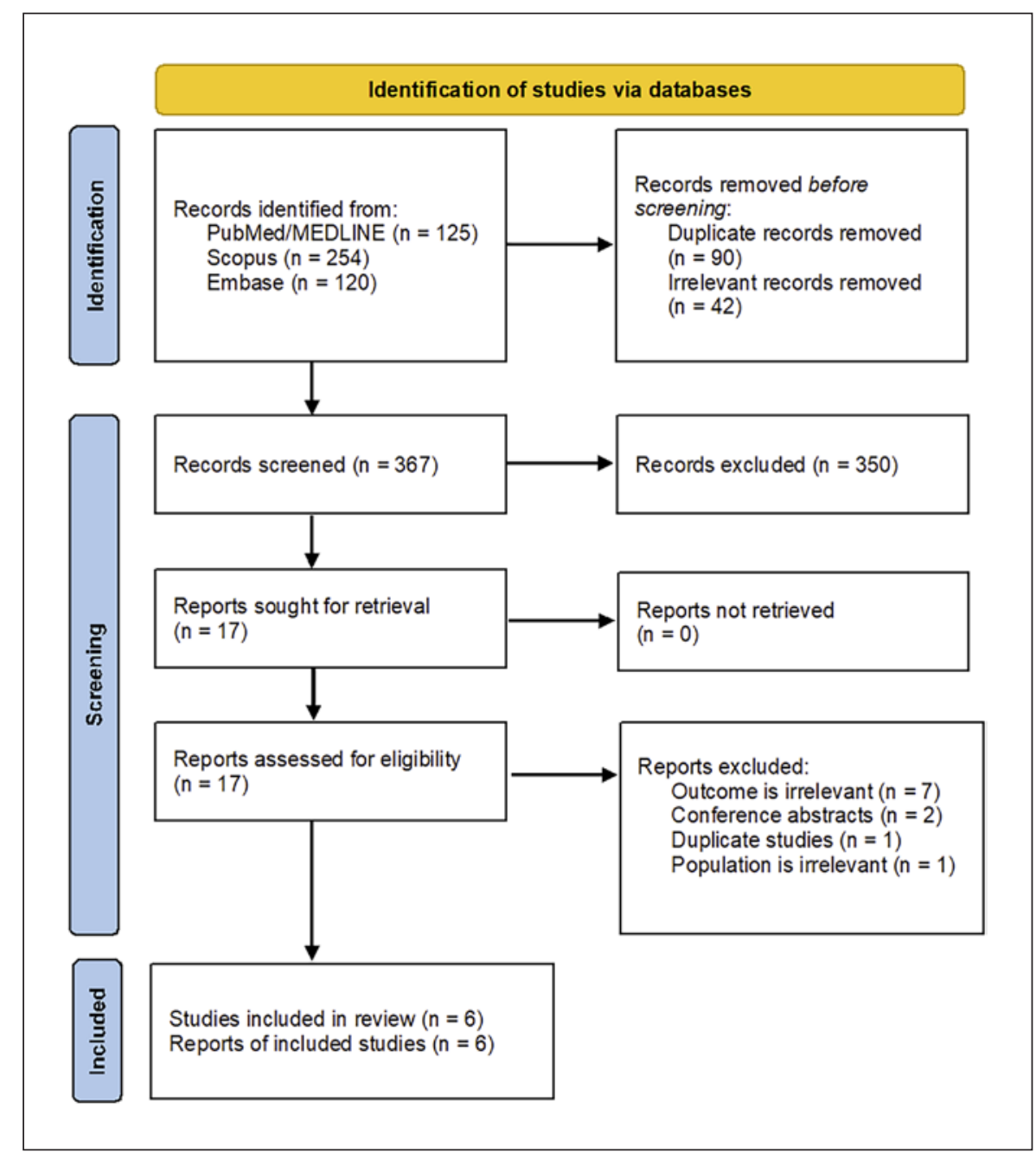

other chronic diseases and environmental factors [2]. This same older population group is also highly prone to experiencing a range of comorbidities and receiving multiple prescription medications. Owing to the various motor and nonmotor symptoms, the treatment of PD often requires the use of a cocktail of specific medications to modify actions on the central nervous system as well as on the enteric and autonomic nervous systems [3].

Polypharmacy typically refers to the use of 5 or more medications at the same time, whereas hyperpolypharmacy is an extreme form of polypharmacy and often constitutes the concomitant use of at least 10 medications on an everyday basis. Polypharmacy or hyperpolypharmacy usually occurs in older patients with multimorbidity and is highly prevalent in frail patients, particularly those who are in long-term care facilities $[4,5]$. Evidence shows that wide ranging prevalences of polypharmacy of $27 \%-59 \%$ and $46 \%-84 \%$ were reported in primary care and hospital settings, respectively, increasing the risk of older adults to receive potentially inappropriate medications [6].

Although prescribing polypharmacy for older adults with complex medical needs could be fairly appropriate [7], polypharmacy and hyperpolypharmacy are considered proxy indicators of inappropriate medication use [8-10]. Furthermore, polypharmacy and hyperpolypharmacy are associated with poor health outcomes like adverse drug events, functional decline, cognitive impairment, and falls, among others [11, 12]. Also, unlike other patient and clinical determinants, polypharmacy or hyperpolypharmacy is considered partly modifiable and suitable to target via appropriate interventions. The need to manage multiple Parkinsonian symptoms and other coexisting conditions using different medications accentuates the need to understand and quantify the extent of polypharmacy in people with $\mathrm{PD}$ to propose practical in- 
terventions such as deprescribing, where applicable. This systematic review and meta-analysis, therefore, presents a comprehensive body of evidence on the prevalence of polypharmacy and hyperpolypharmacy in people with $\mathrm{PD}$ and their association with PD.

\section{Methods}

\section{Search Strategy and Study Selection}

This systematic review and meta-analysis was conducted in accordance with the Preferred Reporting Items for Systematic Review and Meta-analysis guidelines 2020 [13]. A comprehensive literature search was conducted in PubMed/MEDLINE, Scopus, and Embase databases to identify population-based studies from inception to July 2021. Observational studies conducted on the older population with $\mathrm{PD}$, published in English, were searched using a combination of medical subject heading ( $\mathrm{MeSH})$ and non-MeSH terms. A detailed list of MeSH terms and keywords used for each database is enumerated in online supplementary Table 1 (see www. karger.com/doi/10.1159/000521214 for all online suppl. material).

\section{Inclusion and Exclusion Criteria}

We included studies that fulfilled the following criteria:

1. Peer-reviewed research studies conducted in older adults $(\geq 65$ years) with PD.

2. Observational study design.

3. Studies defining polypharmacy as concomitant use of 5 or more medications and hyperpolypharmacy as concomitant use of 10 or more medications.

4. Reported estimates of polypharmacy and hyperpolypharmacy in older patients with PD.

5. Studies reporting the risk of polypharmacy and hyperpolypharmacy in the older population with $\mathrm{PD}$.

Studies were excluded if they (1) did not report estimates of polypharmacy or hyperpolypharmacy in patients with $\mathrm{PD}$; (2) were review articles, case reports or case series, commentaries, conference proceedings, interventional studies, and nonhuman subject studies; (3) did not provide the fundamental definition of polypharmacy or hyperpolypharmacy; (4) were duplicate studies; (5) were studies with unclear definitions, unrelated outcomes measures, or had missing information or insufficient data.

\section{Data Abstraction and Quality Assessment}

Two researchers (A.S.B. and K.V.S.) independently screened the identified studies on the basis of their title and/or abstracts and full text. Retrieved articles were imported to EndNote ${ }^{\mathrm{TM}}$, a literature management software of Thomas Reuters (New York, NY, USA), and duplicates were removed. Disagreement was resolved through consensus, or if necessary, by discussing with an experienced researcher (W.T.). Information on study design, study location, study population, sample size, patient demographics, polypharmacy and hyperpolypharmacy definitions, sample size, estimates of polypharmacy and hyperpolypharmacy, and potential confounders was collected. In addition, we also extracted the risk estimates such as odds ratio (OR), hazard ratio (HR), and risk ratio (RR) with corresponding 95\% confidence intervals (CIs) of the association between polypharmacy/hyperpolypharmacy and risk in older people with $\mathrm{PD}$.

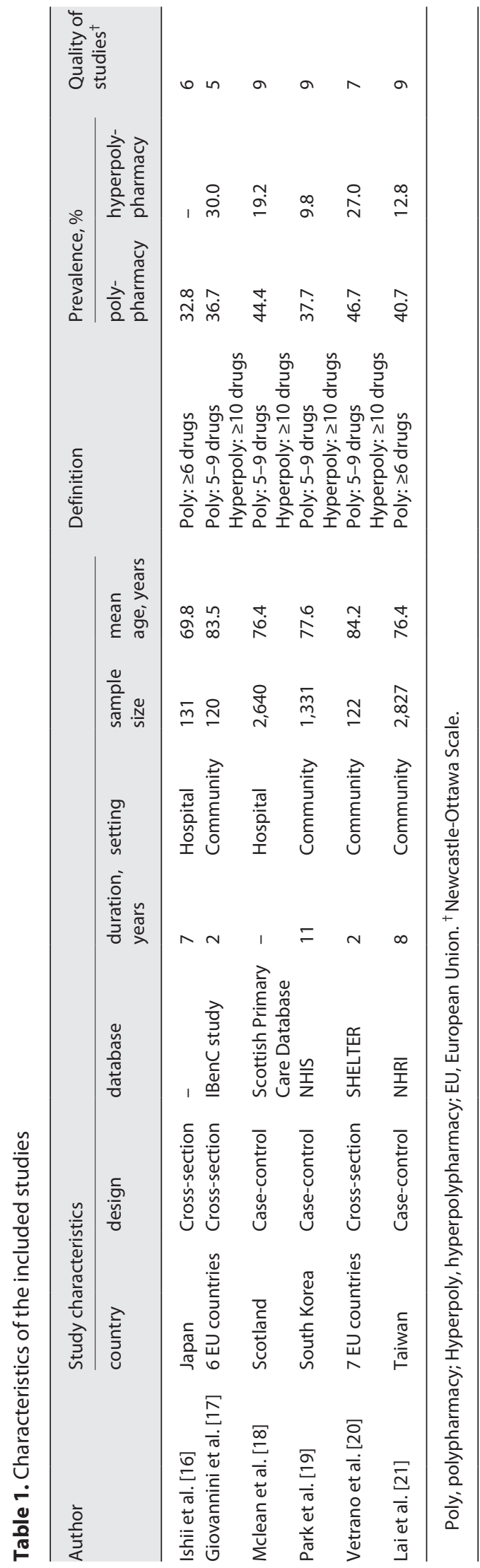

Polypharmacy and Hyperpolypharmacy in Patients with Parkinson's Disease 


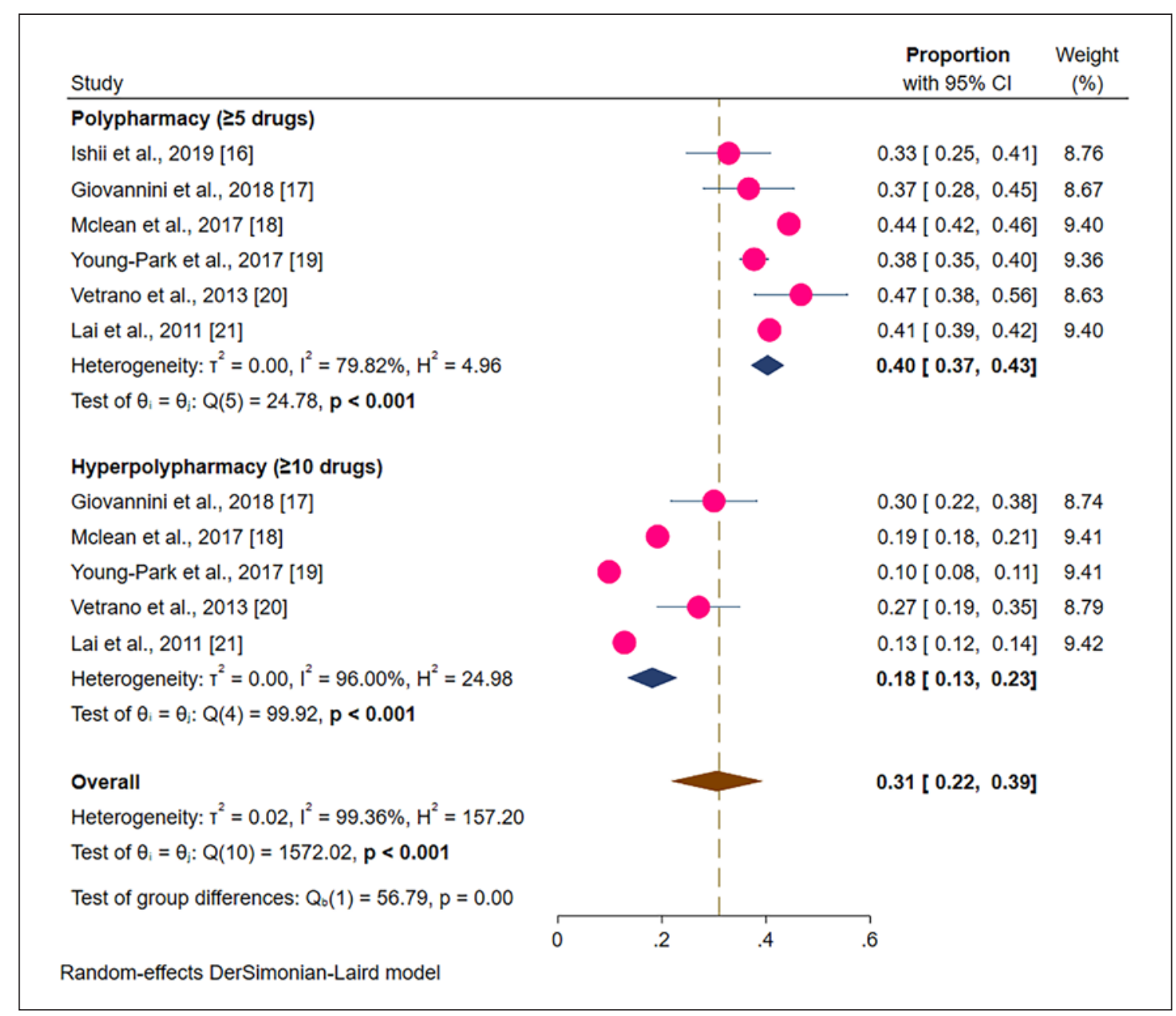

Fig. 2. Prevalence of polypharmacy and hyperpolypharmacy in patients with Parkinson disease [13-18].

The methodological quality of included studies was assessed using the Newcastle-Ottawa Scale (NOS) [14] and graded out of 9 points. The NOS examines 3 major sections: (1) methodological quality of each study (maximum of 5 points were allotted), (2) comparability of the study ( 2 points), and (3) outcomes with related statistical analysis (3 points).

\section{Statistical Analysis}

All statistical analyses were performed using STATA software, version 16.1 MP (StataCorp, College Station, TX, USA). Pooled prevalence was reported as percentages with $95 \%$ CI. Pooled OR was calculated using its log transformation, and inverse variances as weights were calculated for each study to determine the association between polypharmacy/hyperpolypharmacy and risk in PD. Der Simonian and Laird's random-effects model was applied. Statistical heterogeneity was assessed by determining the $I^{2}$ statistics. When $I^{2}$ is $>50 \%$ (indicates high heterogeneity) [15], subgroup analysis and sensitivity analysis were performed to investigate the source of heterogeneity. Publication bias was assessed using funnel plots for symmetry and the Egger's regression and Begg's correlation tests.

\section{Results}

A total of 499 records were identified through several sources. From these, 90 duplicates and 42 irrelevant articles were removed. The remaining 367 records were considered for screening. After screening titles and abstracts, 350 were excluded, and the remaining 17 articles were considered for full-text evaluation. Eleven articles were excluded as their outcome of interest was found irrelevant, insufficient, or ambiguous (online suppl. Table 2). Finally, 6 studies met the inclusion criteria and were included in systematic review and meta-analysis (Fig. 1).

The characteristics of the included studies are summarized in Table 1. In totality, the selected studies comprise 7,171 representative older population with PD [16-21], employed cross-sectional $[16,17,20]$ and case-control $[18,19,21]$ study designs, and were published from 2011 


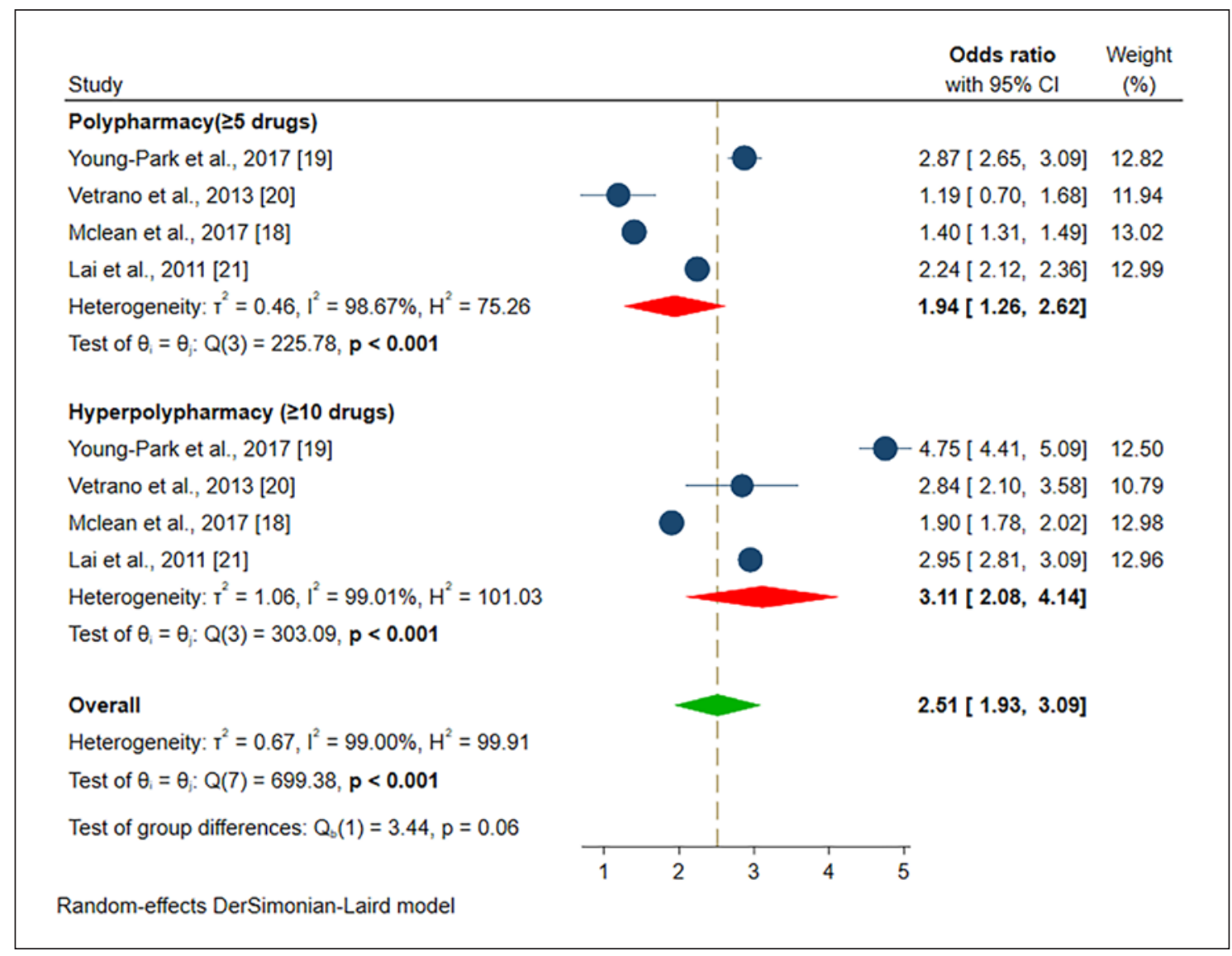

Fig. 3. Risk of polypharmacy and hyperpolypharmacy in patients with Parkinson disease [15-18].

[21] to 2019 [16]. Three studies were conducted in Europe $[17,18,20]$, and one each from Asian countries, namely, Japan [16], South Korea [19], and Taiwan [21]. Four studies were conducted in community settings [17, 19-21], and their sample sizes ranged from 120 [17] to 2,827 [21]. The median age of the study cohort was 78 years and defined polypharmacy as using concomitant use of 5-9 medications [17-20] or over 6 drugs [16, 21] and hyperpolypharmacy as concomitant use of over 10 medications [17-20]. According to the NOS, the average score of studies was 7.5, which indicates high quality, ranging from 5 to 9 (online suppl. Table 3).

\section{Prevalence of Polypharmacy and Hyperpolypharmacy in $P D$}

Six studies comprising 7,171 participants reported a prevalence of polypharmacy and hyperpolypharmacy among older patients with PD [16-21]. The overall prevalence of polypharmacy and hyperpolypharmacy in $\mathrm{PD}$ was found to be $31 \%$ (95\% CI: $22-39$ ). The $I^{2}$ statistics revealed a high degree of heterogeneity across studies $\left(I^{2}=99.3 \%, p<0.001, \tau^{2}=0.02\right)$. The pooled prevalence of polypharmacy in PD was found to be $40 \%$ (95\% CI: $37-43 ; I^{2}=79.8 \%, p<0.001$ ), while the pooled prevalence of hyperpolypharmacy was estimated at $18 \%$ (95\% CI: $\left.13-23 ; I^{2}=96 \%, p<0.001\right)$, more details in Figure 2.

\section{Risk of Polypharmacy and Hyperpolypharmacy in}

\section{Older Patients with $P D$}

A total of 4 studies reported an association between polypharmacy/hyperpolypharmacy and PD in older patients [18-21]. This meta-analysis identified a strong association between polypharmacy/hyperpolypharmacy and PD (OR: 2.51, 95\% CI: 1.93-3.09). There was a high degree of heterogeneity among meta-analyzed studies $\left(I^{2}=99 \%, \tau^{2}=0.67\right)$.

The combined analysis revealed that both polypharmacy (OR: 1.94, 95\% CI: $\left.1.26-2.62 ; I^{2}=98.6 \%, \tau^{2} 0.46\right)$ and hyperpolypharmacy (OR: 3.11, 95\% CI: 2.08-4.14, $\left.I^{2}=99 \%, \tau^{2} 1.06\right)$ are independent risk factors for PD. The 
Table 2. Stratified meta-analysis of the prevalence of polypharmacy and hyperpolypharmacy in Parkinson's disease

\begin{tabular}{|c|c|c|c|c|c|c|c|}
\hline \multirow[t]{2}{*}{ Characteristics } & \multirow{2}{*}{$\begin{array}{l}\text { Studies, } \\
n\end{array}$} & \multirow{2}{*}{$\begin{array}{l}\text { Pooled prevalence } \\
\text { in percentage } \\
(95 \% \mathrm{Cl})\end{array}$} & \multirow[t]{2}{*}{$R^{2}, \%$} & \multirow[t]{2}{*}{$Z$} & \multicolumn{3}{|c|}{ Heterogeneity between groups } \\
\hline & & & & & $Q$ & df & $p$ value \\
\hline \multicolumn{8}{|l|}{ Polypharmacy } \\
\hline \multicolumn{8}{|l|}{ Year of publication } \\
\hline Before 2015 & 2 & $41(39-43)$ & - & 45.2 & 0.58 & 1 & 0.447 \\
\hline After 2015 & 4 & $39(33-44)$ & 86.8 & 14.3 & & & \\
\hline \multicolumn{8}{|l|}{ Study design } \\
\hline Cross-sectional & 3 & $39(31-47)$ & - & 22.4 & 0.29 & 1 & 0.59 \\
\hline Case-control & 3 & $41(37-45)$ & - & 9.4 & & & \\
\hline \multicolumn{8}{|l|}{ Study location } \\
\hline Asia & 3 & $39(35-42)$ & - & 23.6 & 3.12 & 1 & 0.080 \\
\hline Europe & 3 & $43(39-48)$ & - & 19.6 & & & \\
\hline \multicolumn{8}{|l|}{ Study duration } \\
\hline$\leq 2$ years & 2 & $42(35-48)$ & - & 13.2 & 9.80 & 2 & 0.01 \\
\hline$>2$ years & 3 & $39(35-42)$ & - & 45.9 & & & \\
\hline \multicolumn{8}{|l|}{ Study center } \\
\hline Single & 2 & $44(42-46)$ & - & 46.5 & 5.76 & 1 & 0.02 \\
\hline Multiple & 4 & $40(37-42)$ & 51.7 & 28.1 & & & \\
\hline \multicolumn{8}{|l|}{ Setting } \\
\hline Community & 4 & $40(37-42)$ & 51.7 & 28.1 & 5.76 & 1 & 0.02 \\
\hline Hospital & 2 & $44(42-46)$ & - & 46.5 & & & \\
\hline \multicolumn{8}{|l|}{ Sample size } \\
\hline$<1,000$ & 3 & $39(31-47)$ & - & 9.3 & 0.29 & 1 & 0.59 \\
\hline$>1,000$ & 3 & $41(37-45)$ & - & 22.4 & & & \\
\hline \multicolumn{8}{|l|}{ Quality of studies* } \\
\hline High $(\geq 7)$ & 4 & $42(38-45)$ & 84.3 & 24.2 & 4.08 & 1 & 0.04 \\
\hline $\operatorname{Low}(<7)$ & 2 & $35(29-40)$ & - & 11.5 & & & \\
\hline \multicolumn{8}{|l|}{ Hyperpolypharmacy } \\
\hline \multicolumn{8}{|l|}{ Year of publication } \\
\hline Before 2015 & 2 & $13(12-14)$ & - & 21.1 & 1.71 & 1 & 0.19 \\
\hline After 2015 & 3 & $19(10-27)$ & - & 4.4 & & & \\
\hline \multicolumn{8}{|l|}{ Study design } \\
\hline Cross-sectional & 2 & $28(23-34)$ & - & 9.8 & 13.95 & 1 & $<0.01$ \\
\hline Case-control & 3 & $14(9-19)$ & - & 5.3 & & & \\
\hline \multicolumn{8}{|l|}{ Study location } \\
\hline Asia & 2 & $12(11-13)$ & - & 23.5 & 10.9 & 1 & 0.01 \\
\hline Europe & 3 & $25(17-32)$ & - & 6.3 & & & \\
\hline Study duration & & & & & & & \\
\hline$\leq 2$ years & 2 & $28(23-34)$ & - & 9.8 & 91.4 & 2 & $<0.01$ \\
\hline$>2$ years & 2 & $12(11-13)$ & - & 23.5 & & & \\
\hline Study center & & & & & & & \\
\hline Single & 1 & $19(18-21)$ & - & 25.0 & 0.50 & 1 & 0.48 \\
\hline Multiple & 4 & $17(12-22)$ & 92.6 & 6.9 & & & \\
\hline Setting & & & & & & & \\
\hline Community & 1 & $19(18-21)$ & 92.6 & 6.9 & 0.50 & 1 & 0.48 \\
\hline Hospital & 4 & $17(12-22)$ & - & 25.0 & & & \\
\hline Sample size & & & & & & & \\
\hline$<1,000$ & 2 & $28(23-34)$ & - & 9.8 & 13.9 & 1 & $<0.01$ \\
\hline$>1,000$ & 3 & $14(9-19)$ & - & 5.3 & & & \\
\hline Quality of studies* & & & & & & & \\
\hline $\operatorname{High}(\geq 7)$ & 4 & $16(11-21)$ & 96.4 & 6.4 & 8.1 & 1 & $<0.01$ \\
\hline Low $(<7)$ & 1 & $30(23-39)$ & - & 7.1 & & & \\
\hline
\end{tabular}

* Newcastle-Ottawa Scale. 
Table 3. Subgroup analysis of polypharmacy and hyperpolypharmacy and risk in Parkinson's disease

\begin{tabular}{|c|c|c|c|c|c|c|c|c|}
\hline \multirow[t]{2}{*}{ Subgroup } & \multicolumn{4}{|c|}{ Polypharmacy } & \multicolumn{4}{|c|}{ Hyperpolypharmacy } \\
\hline & $\begin{array}{l}\text { studies, } \\
n\end{array}$ & $\begin{array}{l}\text { odds ratio } \\
(95 \% \mathrm{Cl})\end{array}$ & $\begin{array}{l}P^{2} \\
\%\end{array}$ & $p$ value & $\begin{array}{l}\text { studies, } \\
n\end{array}$ & $\begin{array}{l}\text { odds ratio } \\
(95 \% \mathrm{Cl})\end{array}$ & $R^{2}, \%$ & $p$ value \\
\hline \multicolumn{9}{|l|}{ Study design } \\
\hline Cross-sectional & 1 & 1.19 (0.73-1.94) & - & 0.488 & 1 & $2.84(1.36-5.93)$ & 94.8 & 0.005 \\
\hline Case-control & 5 & $2.06(1.37-3.11)$ & 96.7 & 0.001 & 3 & 2.91 (1.89-4.47) & - & $<0.001$ \\
\hline \multicolumn{9}{|l|}{ Countries } \\
\hline Asia & 2 & $1.77(1.12-2.80)$ & 97.4 & 0.015 & 2 & $2.37(1.54-3.64)$ & 95.4 & $<0.001$ \\
\hline Europe & 2 & $1.90(0.80-4.50)$ & 90.2 & 0.144 & 2 & 4.09 (2.59-6.47) & 35.6 & $<0.001$ \\
\hline \multicolumn{9}{|l|}{ Sample size } \\
\hline$<1,000$ & 1 & $1.19(0.73-1.94)$ & 0.0 & 0.488 & 1 & $2.84(1.36-5.93)$ & - & $<0.001$ \\
\hline$>1,000$ & 3 & $2.06(1.37-3.11)$ & 96.7 & 0.001 & 3 & $2.91(1.89-4.47)$ & 94.8 & 0.005 \\
\hline \multicolumn{9}{|l|}{ Setting } \\
\hline Community & 3 & 1.61 (1.09-2.38) & 95.0 & 0.018 & 3 & $2.44(1.67-3.56)$ & 91.0 & $<0.001$ \\
\hline Hospital & 1 & $2.87(2.30-3.58)$ & - & $<0.001$ & 1 & $4.75(3.39-6.66)$ & - & $<0.001$ \\
\hline \multicolumn{9}{|l|}{ Study duration } \\
\hline$\leq 2$ years & 1 & $1.19(0.73-1.94)$ & - & 0.488 & 1 & $2.84(1.36-5.93)$ & - & 0.005 \\
\hline$>2$ years & 2 & $1.77(1.12-2.80)$ & 97.4 & 0.015 & 2 & $2.37(1.54-3.64)$ & 95.4 & $<0.001$ \\
\hline
\end{tabular}

risk associated with the disease increased by $117 \%$ when the number of medications increased from 5 to $>10$. More details regarding these observations are presented in Figure 3.

\section{Stratified Analysis}

A stratified meta-analysis of the prevalence of polypharmacy and hyperpolypharmacy in the older PD population is summarized in Table 2. We stratified the studies by various baseline characteristics and investigated the sources of heterogeneity and differences between the groups. Significant heterogeneity was detected among certain subgroups; for instance, studies with short duration ( $\leq 2$ years) had a higher pooled prevalence of polypharmacy (42\%) compared to those studies conducted for $>2$ years $(39 \%)$. A significant heterogeneity between the groups was observed $(Q=9.80, \mathrm{df}=2, p=0.01)$. Similar differences were observed in stratified analysis based on study center (single vs. multiple), type of settings (community vs. hospital), and quality of studies based on NOS (high vs. low). With regard to hyperpolypharmacy, significant differences were noticed across groups (study design, study location, study duration, sample size, and quality of studies). More details are encapsulated in Table 2.

\section{Subgroup Analyses}

Subgroup analyses was performed to identify potential sources of heterogeneity. The test of heterogeneity confirmed that variations in the results across the polyphar- macy and hyperpolypharmacy groups were not solely due to chance. This is because studies were conducted in different countries and had different study designs, sample size, study settings, and study duration. The detailed results of subgroup analyses of polypharmacy and hyperpolypharmacy in older patients with $\mathrm{PD}$ are summarized in Table 3.

\section{Publication Bias and Sensitivity Analysis}

Visual examination of the funnel plots confirmed asymmetry and suggested publication bias (online suppl. Fig. 1a, b). However, Egger's regression and Begg's correlation tests evidenced no significant publication bias on studies reporting polypharmacy (Egger's $p=0.519$ and Begg's $p=0.851$ ) and hyperpolypharmacy (Egger's $p=$ 0.394 and Begg's $p=0.624$ ). Sensitivity analysis was performed to appraise each study's influence on overall meta-analysis and revealed that no individual study contributed greatly to the heterogeneity of the estimates and did not exhibit significant differences beyond the 95\% CI limit (online suppl. Fig. 1c, d).

\section{Discussion}

Medication-related problems are a particular concern in older patients. Concomitant use of multiple medications to treat Parkinsonian symptoms increases the risk 
of potential drug-drug interactions and the probability of receiving inappropriate medications. This review examined the prevalence of polypharmacy, which is the concomitant use of 5-9 medications, and hyperpolypharmacy (10 or more medications), and its association with PD in older adults.

The pooled analyses revealed that two out of five and one in five PD patients were exposed to polypharmacy and hyperpolypharmacy, respectively. Although reported prevalence of polypharmacy varies from $10 \%$ to $90 \%$, our review reveals a higher prevalence compared with previously reported polypharmacy prevalences of $32 \%, 36 \%$, and 39\% among general older populations across Europe [22], Australia [23], and the USA [24], respectively. The prevalence of hyperpolypharmacy we found is similar to the $20 \%$ prevalence reported in older adults aged 70-74 years from the European Union [25], while a higher proportion of $40 \%$ was reported in the USA residential cares [26]. Much higher proportions of polypharmacy and hyperpolypharmacy were also reported in other chronic conditions, such as older adults with heart failure (95\% and $55 \%$, respectively) [27] and cancer ( $41 \%$ and $43 \%$, respectively) [28]. One study targeting older adults with Alzheimer dementia, on the other hand, reported that up to a third of participants were on polypharmacy [29].

Our review showed that having multiple medications was not only associated with increased risk of PD, but also the risk increased by $117 \%$ when the number of medications used increased from between 5 and 9 medications to $>10$ per day. Additionally, the increase in the categorical threshold from 5 or more to 10 or more led to an increase in the risk of PD from $94 \%$ to $211 \%$. The findings agree with previous individual studies that found that polypharmacy and hyperpolypharmacy are independent risk factors for PD [18-21]. This is attributed largely to the wide range of comorbidities as well as neurological and nonneurological symptoms that occur in people with $\mathrm{PD}$ [30], which, in turn, necessitate the use of multiple medications. The nonmotor symptoms commonly reported in $\mathrm{PD}$, such as cognitive and neuropsychiatric disturbances, are particularly important symptoms that often expand the need to use pharmacologic treatments beyond those that act via the dopaminergic pathway [31]. Additionally, nonmotor chronic conditions seen in people with PD, including diabetes, cancer, hypertension, and depression, require long-term use of multiple medications [30], further increasing the number of medications used in PD.

To the best of our knowledge, this is the first and the most comprehensive systematic review on the prevalence and association of polypharmacy and hyperpolypharma- cy with PD in older patients. A recent systematic review and meta-analysis by $\mathrm{Chen}$ and $\mathrm{Yu}$ [32], which included 8 studies on the association between polypharmacy and PD prevalence, did not provide a comprehensive picture of the polypharmacy and hyperpolypharmacy risk in PD patients. In contrast, categorization of included studies using the number of medications exceeding a specific threshold to define the exposure made our results reliable and consistent with previous studies conducted on the older population with cognitive impairment [33, 34], frailty $[4,35]$, and dementia $[36,37]$. This categorization also makes it more convenient for developing practical interventions targeted at people at a higher risk of deterioration in clinical outcomes.

Polypharmacy is deemed an important cause of inappropriate medication use in older populations with PD [38]. A narrative review on this issue has been published by Csoti et al. [3], giving an overview of various pharmacotherapeutic strategies in treating PD patients. The authors extensively discussed the most common challenges faced by PD patients with multiple morbidities. In this study, we incorporated 4 observational studies comprising 7,171 participants and used maximum-adjusted estimates to report the association between multiple medication use and PD. Altogether, the results of our meta-analysis showed a significantly higher probability of polypharmacy and hyperpolypharmacy users compared to unexposed or controls. However, notable heterogeneity was found in our meta-analysis. Our findings complement a previously published review that concluded the need for a specific clinical tool to monitor and optimize pharmacotherapy in $\mathrm{PD}$ patients [3].

Although our findings are noteworthy, because of the use of case-control and cross-sectional studies and a lack of prospective cohort research on this topic, it is difficult to assert with confidence the association between polypharmacy/hyperpolypharmacy and PD at a clinically significant magnitude. Because of the observational nature of included studies, high heterogeneity, and specific methodological flaws thereof, it is not also possible to establish that polypharmacy/hyperpolypharmacy was definitely associated with PD. Both stratified and subgroup analyses identified several significant factors that contribute to high heterogeneity. However, given the observational nature of studies, it limits our ability to establish respective causal relationships of polypharmacy and hyperpolypharmacy with PD. Moreover, there is a strong caution that clinicians should be aware of the potential risk of polypharmacy while treating patients with PD. Whenever feasible, clinicians should apply evidence- 
based explicit criteria and deprescribing strategies to ensure optimal use of multiple medication in PD patients.

\section{Strengths and Limitations}

The present meta-analysis has several strengths and a few limitations. This systematic review provided comprehensive, up-to-date information, and detailed evaluation of the prevalence and risk of polypharmacy and hyperpolypharmacy in older PD patients. This is the first meta-analysis in the scientific literature published on this topic and furnished clearer insights into the relationship between multiple medication use and PD by including 6 studies targeting over 7,000 PD older patients. Furthermore, estimates are summarized by percentages and ORs using random-effect meta-analysis to identify the prevalence and risk contributing to polypharmacy/hyperpolypharmacy exposure in the $\mathrm{PD}$ population by using maximum-adjusted estimates. The assessment of study quality by the NOS indicated that most of the included studies were of superior quality, which implied minimal risk of bias and comparable cases and controls. Egger's regression and Begg's correlation tests evidenced no significant publication bias on studies. However, we observed asymmetry in funnel plot distribution and acknowledge the concern of publication bias. Furthermore, the sensitivity analysis indicated no significant differences, beyond the limits of $95 \%$ CI on overall outcomes, suggesting that their inclusion was justified and our results are reliable. Despite these strengths, we acknowledge that our study was limited by the number of studies, case-control and cross-sectional design, and small sample size that reported the prevalence and risk of polypharmacy/hyperpolypharmacy in older PD patients. $\mathrm{Nu}$ merous comorbidities that are known to be associated with $\mathrm{PD}$, such as diabetes, depression, anemia, cancer, hypertension, sleep disorders, cognitive impairment, hyposmia, constipation, and restless leg syndrome [32], were also not adjusted for in most studies.

Moreover, Giovannini et al. [17] and Vetrano et al. [20] provided information on polypharmacy and PD. However, their primary objectives differed from ours. Nevertheless, given that the included studies were conducted in Europe and Asia, caution should be taken while generalizing our findings to other contexts.

\section{Conclusion}

This meta-analysis summarized the extent of polypharmacy and hyperpolypharmacy specific to older adults with PD. With a rising trend of polypharmacy and hyperpolypharmacy over the past decade, two out of five and one in five PD patients are exposed to polypharmacy and hyperpolypharmacy, respectively. Older PD patients had a higher probability and are at an increased risk of polypharmacy and hyperpolypharmacy that can potentially aggravate or interact with neurological and psychological symptoms. Therefore, while prescribing, clinicians should take polypharmacy into account, and medication review in older PD adults is important to improve the quality of prescription. These steps may eventually reduce the risk of inappropriate medication use and drugrelated problems. Moreover, more emphasis is needed to understand the pestilent phenomenon of polypharmacy and hyperpolypharmacy in older PD patients.

\section{Statement of Ethics}

An ethics statement was not required for this study type, as no human or animal subjects or materials were used.

\section{Conflict of Interest Statement}

The authors declared no competing interest for this work.

\section{Funding Sources}

Research works of Assoc. Prof. Fialová and her research team were supported by research projects: EuroAgeism H2020 MSCFITN-764632, Inomed NO.CZ.02.1.01/0.0/0.0/18_069/0010046, Progress Q42 Faculty of Pharmacy, Charles University (research group "Ageing, Polypharmacy and Changes in the Therapeutic value of Drugs in the AgeD"), START/MED/093CZ.02.2.69/ 0.0/0.0/19_073/0016935, SVV 260551, and I-CARE4OLD H2020965341 projects.

\section{Author Contributions}

A.S.B. is the principal investigator of the study; A.S.B. and K.V.S. contributed to data providing and analysis; A.S.B., K.V.S., and W.T. contributed to literature review; A.S.B. and W.T. contributed to writing; A.S.B., W.T., and D.F. contributed to manuscript revision. All authors read and approved the final manuscript.

\section{Data Availability Statement}

All data generated or analyzed during this study are included in this article and/or its online supplementary material files. Further enquiries can be directed to the corresponding author.
Polypharmacy and Hyperpolypharmacy in Patients with Parkinson's Disease 


\section{References}

1 Lees AJ, Hardy J, Revesz T. Parkinson's disease. Lancet. 2009;373(9680):2055-66.

2 GBD 2016 Parkinson's Disease Collaborators. Global, regional, and national burden of Parkinson's disease, 1990-2016: a systematic analysis for the Global Burden of Disease Study 2016. Lancet Neurol. 2018;17(11):93953.

3 Csoti I, Herbst H, Urban P, Woitalla D, Wüllner U. Polypharmacy in Parkinson's disease: risks and benefits with little evidence. J Neural Transm. 2019;126(7):871-8.

4 Palmer K, Villani ER, Vetrano DL, Cherubini A, Cruz-Jentoft AJ, Curtin D, et al. Association of polypharmacy and hyperpolypharmacy with frailty states: a systematic review and meta-analysis. Eur Geriatr Med. 2019;10(1): 9-36.

5 Jokanovic N, Tan EC, Dooley MJ, Kirkpatrick CM, Bell JS. Prevalence and factors associated with polypharmacy in long-term care facilities: a systematic review. J Am Med Dir Assoc. 2015;16(6):535.e1-12.

6 Elmståhl S, Linder H. Polypharmacy and inappropriate drug use among older people: a systematic review. Healthy Aging Clin Care Elderly. 2013;5:1

7 Cadogan CA, Ryan C, Hughes CM. Appropriate polypharmacy and medicine safety: when many is not too many. Drug Saf. 2016;39(2): 109-16.

8 Poudel A, Hubbard RE, Nissen L, Mitchell C. Frailty: a key indicator to minimize inappropriate medication in older people. QJM. 2013; 106(10):969-75.

9 Jetha S. Polypharmacy, the elderly, and deprescribing. Consult Pharm. 2015;30(9):527-32.

10 Guillot J, Maumus-Robert S, Marceron A, Noize P, Pariente A, Bezin J. The burden of potentially inappropriate medications in chronic polypharmacy. J Clin Med. 2020; 9(11):3728

11 Maher RL, Hanlon J, Hajjar ER. Clinical consequences of polypharmacy in elderly. Expert Opin Drug Saf. 2014;13(1):57-65.

12 Frazier SC. Health outcomes and polypharmacy in elderly individuals: an integrated literature review. J Gerontol Nurs. 2005;31(9): 4-11.

13 Page MJ, McKenzie JE, Bossuyt PM, Boutron I, Hoffmann TC, Mulrow CD, et al. The PRISMA 2020 statement: an updated guideline for reporting systematic reviews. BMJ. 2021;372: n71.

14 Wells GA, Shea B, O'Connell D, Peterson J, Welch V, Losos M, et al. The Newcastle-Ottawa Scale (NOS) for assessing the quality of nonrandomised studies in meta-analyses. Oxford; 2000
15 Higgins JP, Thompson SG. Quantifying heterogeneity in a meta-analysis. Stat Med. 2002; 21(11):1539-58.

16 Ishii N, Mochizuki H, Sakai K, Ogawa G, Shiomi K, Nakazato M. Polypharmacy associated with cognitive decline in newly diagnosed Parkinson's disease: a Cross-Sectional Study. Dement Geriatr Cogn Dis Extra. 2019; 9(3):338-43.

17 Giovannini S, van der Roest HG, Carfi A, Finne-Soveri H, Garms-Homolová V, Declercq A, et al. Polypharmacy in home care in Europe: cross-sectional data from the IBenC Study. Drugs Aging. 2018;35(2):145-52.

18 McLean G, Hindle JV, Guthrie B, Mercer SW. Co-morbidity and polypharmacy in Parkinson's disease: insights from a large Scottish primary care database. BMC Neurol. 2017; 17(1):126.

19 Park HY, Park JW, Sohn HS, Kwon JW. Association of Parkinsonism or Parkinson disease with polypharmacy in the year preceding diagnosis: a Nested Case-Control Study in South Korea. Drug Saf. 2017;40(11):1109-18.

20 Vetrano DL, Tosato M, Colloca G, Topinkova E, Fialova D, Gindin J, et al. Polypharmacy in nursing home residents with severe cognitive impairment: results from the SHELTER Study. Alzheimers Dement. 2013;9(5):58793.

21 Lai SW, Su LT, Lin CH, Tsai CH, Sung FC, Hsieh DP, et al. Polypharmacy increases the risk of Parkinson's disease in older people in Taiwan: a population-based study. Psychogeriatrics. 2011;11(3):150-6.

22 Midão L, Giardini A, Menditto E, Kardas P, Costa E. Polypharmacy prevalence among older adults based on the survey of health, ageing and retirement in Europe. Arc Gerontol Geriatr. 2018;78:213-20.

23 Page AT, Falster MO, Litchfield M, Pearson SA, Etherton-Beer C. Polypharmacy among older Australians, 2006-2017: a population based study. Med J Australia. 2019;211(2):715.

24 Charlesworth CJ, Smit E, Lee DS, Alramadhan F, Odden MC. Polypharmacy among adults aged 65 years and older in the United States: 1988-2010. J Gerontol A Biol Sci Med Sci. 2015;70(8):989-95.

25 Mair A, Fernandez-Llimos F, Alonso A, Harrison C, Hurding S, Kempen T, et al. Polypharmacy management by 2030: a patient safety challenge; 2017.

26 Dwyer LL, Han B, Woodwell DA, Rechtsteiner EA. Polypharmacy in nursing home residents in the United States: results of the 2004 National Nursing Home Survey. Am J Geriatr Pharmacother. 2010;8(1):63-72.
27 Unlu O, Levitan EB, Reshetnyak E, KneifatiHayek J, Diaz I, Archambault A, et al. Polypharmacy in older adults hospitalized for heart failure. Circ Heart Fail. 2020;13(11): e006977.

28 Nightingale G, Hajjar E, Swartz K, AndrelSendecki J, Chapman A, et al. Evaluation of a pharmacist-led medication assessment used to identify prevalence of and associations with polypharmacy and potentially inappropriate medication use among ambulatory senior adults with cancer. J Clin Oncol. 2015;33(13): 1453-9.

29 de Souza PM, Kusano LTE, Kusano LTE, dos Santos Neto LL. Prevalência da polifarmácia quantitativa e qualitativa em idosos com demência de alzheimer. Geriatr Gerontol Aging. 2018;12(3):143-7.

30 Santiago JA, Bottero V, Potashkin JA. Biological and clinical implications of comorbidities in Parkinson's disease. Front Aging Neurosci. 2017;9:394.

31 Park A, Stacy M. Non-motor symptoms in Parkinson's disease. J Neurol. 2009;256(Suppl 3):293-8.

32 Chen Y, Yu Z. Association of polypharmacy and Parkinson's disease prevalence. Bratisl Lek Listy. 2021;122(2):158-60.

33 Umegaki H, Yanagawa M, Komiya H, Matsubara M, Fujisawa C, Suzuki Y, et al. Polypharmacy and gait speed in individuals with mild cognitive impairment. Geriatr Gerontol Int. 2019;19(8):730-5.

34 Fabbietti P, Ruggiero C, Sganga F, Fusco S, Mammarella F, Barbini N, et al. Effects of hyperpolypharmacy and potentially inappropriate medications (PIMs) on functional decline in older patients discharged from acute care hospitals. Arch Gerontol Geriatr. 2018;77: 158-62.

35 Moon JH, Huh JS, Won CW, Kim HJ. Is polypharmacy associated with cognitive frailty in the elderly? results from the Korean Frailty and Aging Cohort Study. J Nutr Health Aging. 2019;23(10):958-65.

36 Leelakanok N, D’Cunha RR. Association between polypharmacy and dementia: a systematic review and metaanalysis. Aging Ment Health. 2019;23(8):932-41.

37 Jester DJ, Molinari V, Zgibor JC, Volicer L. Prevalence of psychotropic polypharmacy in nursing home residents with dementia: a meta-analysis. Int Psychogeriatr. 2021;33: $1-16$.

38 Yusupov E, Chen D, Krishnamachari B. Medication use and falls: applying Beers criteria to medication review in Parkinson's disease. SAGE Open Med. 2017; 5: 2050312117743673. 